\title{
An Ultra-Short InP Nanowire Laser Monolithic Integrated on (001) Silicon Substrate
}

\author{
Zhechao Wang ${ }^{1,2}$, Bin $\operatorname{Tian}^{1,2}$, Mohan Paladugu ${ }^{3}$, Marianna Pantouvaki ${ }^{3}$, Clement Merckling $^{3}$, Weiming Guo ${ }^{3}$, Johan \\ Dekoster $^{3}$, Matty Caymax ${ }^{3}$, Joris Van Campenhout ${ }^{3}$, Philippe Absil ${ }^{3}$, Dries Van Thourhout ${ }^{1,2}$ \\ 1. INTEC-department, Ghent University-IMEC, Sint-Pietersnieuwstraat 41, Ghent 9000, Belgium \\ 2. Center for Nano- and Biophotonics (NB-Photonics), Ghent University, Sint-Pietersnieuwstraat 41, Ghent 9000, \\ Belgium \\ 3. IMEC, Kapeldreef 753001 Heverlee, Belgium
}

Silicon photonics holds the promise of converging electronics and photonics. The key component, a lowcost high-performance laser, is still missing however within this platform. Although novel solutions have been proposed to increase the light emission directly from silicon (or Ge), compared with their III-V counterparts [1-2], these solutions are still in their infancy. Recently, the epitaxial growth of III-Vs on silicon regained a wide interest. III-V nanowire growth has been widely investigated. However, most of the III-V nanowire lasers on silicon require a complex cleaving and transfer process, which make these devices not suitable for dense integration [3]. In addition, the large cavity dimensions along the nanowire axis (several microns) hinder dense integration. Here, we present the first room-temperature operation of an ultra-short InP nanowire laser that is epitaxially grown on an exactly [001] oriented silicon substrate. The sub-micron sized laser cavity largely enhances the interaction of the lasing mode with the gain medium, and a large spontaneous emission factor has been obtained.

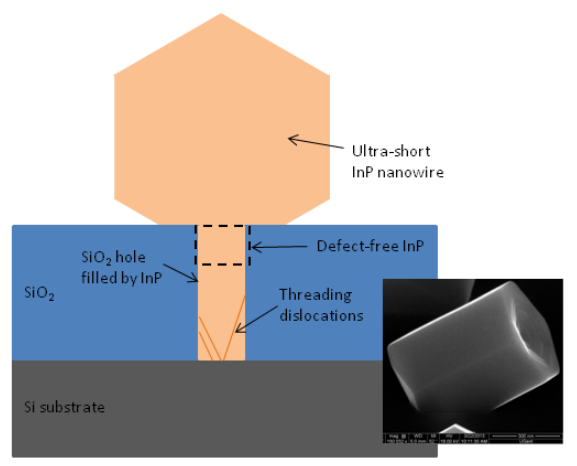

Fig. 1 Schematic cross-section view of the short InP/Si nanowire cavity. Insert: $45^{\circ}$ titled SEM view of the InP/Si nanowire cavity

To obtain these results, we developed a unique epitaxial process which enlarges the lateral dimensions of a defect-free InP/Si nanowire such that it can sustain high quality optical modes. As shown in Fig.1, as the first step, holes of $100 \mathrm{~nm}$ diameter are defined in a $\mathrm{SiO}_{2}$ mask to expose the silicon surface. After depositing and annealing a layer of $\mathrm{Ge}$, epitaxial growth of InP was carried out in a metal organic chemical vapor deposition (MOCVD) reactor. The defect necking effect [4] of the narrow holes blocks propagation of the dislocations from the lattice-mismatched interface and defect-free InP is obtained at the mask surface. On this virtual lattice matched substrate, InP nanowires are grown along the [111] direction. Lateral overgrowth of $\mathrm{InP}$ on the $\mathrm{SiO}_{2}$ mask took place right after the beginning of the nanowire growth, therefore the dimension of the formed nanowire cavity is much larger than the $\mathrm{SiO}_{2}$ hole. The insert of Fig. 1 shows a $45^{\circ}$ titled scanning electron microscope (SEM) picture of a typical InP nanowire on silicon. The length of the nanowire cavity is only $700 \mathrm{~nm}$. 
Thanks to the limited dimensions of the $\mathrm{SiO}_{2}$ hole that connects the cavity and the substrate, the downwards leakage loss is low enough to permit room-temperature laser oscillation. A Nd:YAG laser with 7 nanoseconds pulsed output is used as the optical pump source. Fig.2(a) shows the light in-light out (L-L) curve measured in a photoluminescence (PL) setup. The PL spectrum recorded above threshold is also shown in the insert. The clear slope transition in the L-L curve is a strong signature of threshold behavior. By applying a classic rate equation fitting process, a large spontaneous factor of 0.1 is obtained, which indicates very compact optical confinement. In order to identify the optical mode that oscillates in the short nanowire cavity, a finite-difference time-domain (FDTD) based numerical tool (Lumerical) was used, and the simulated optical mode distributions are plotted in Fig.2(b) and (c). As one can expect, regular Fabry-Perot modes, which are found in most of the demonstrated nanowire lasers, cannot oscillate in this cavity due to the irregular bottom contact with the substrate. Instead, a helically propagating cavity mode with hexagonal whispering gallery-like mode pattern in the transverse plane is found by simulations [5]. Its insensitivity to the bottom contact helps to maintain a relative high-Q factor for the lasing mode ( 400 in this case). The asymmetrical mode profile shown in Fig.2(c) is mainly caused by the presence of the InP-filled $\mathrm{SiO}_{2}$ trench at the bottom.
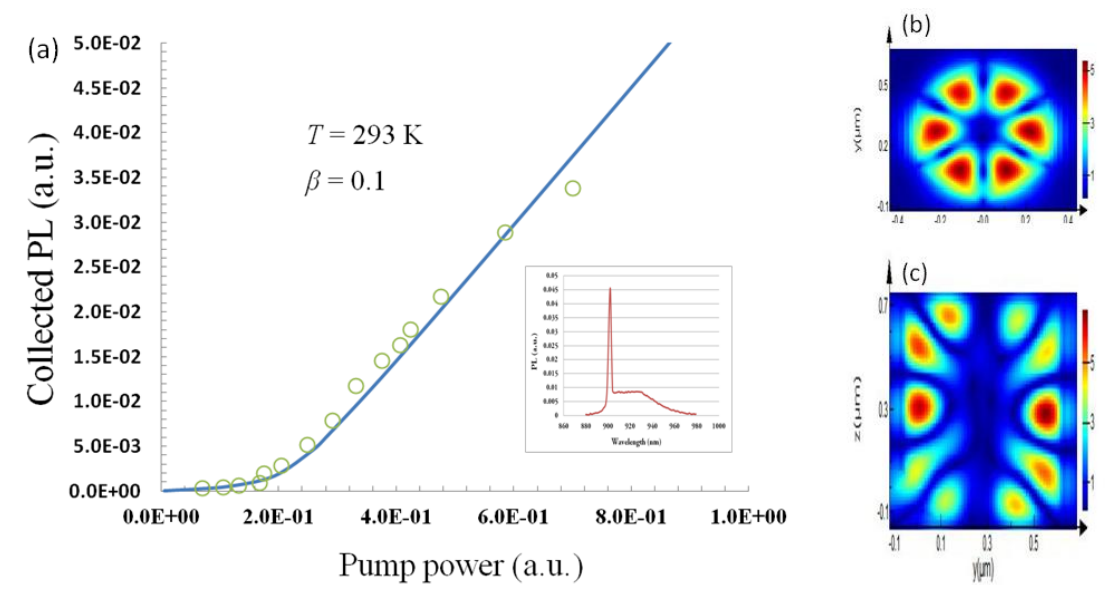

Fig. 1 (a) Mapping of the PL emission integrated around the lasing wavelength with respect to the pump power intensity. Insert: PL spectrum recorded above threshold. (b) \& (c) FDTD simulated lasing mode profile in the transverse plane and the cross section plane along the wire axis.

In conclusion, an ultra-short InP nanowire laser with compact mode size was epitaxially grown on a (001) silicon substrate. Optically pumped room-temperature lasing was achieved. This monolithic integrated micro-laser is very promising for applications that require high integration density.

\section{Acknowledgement:}

We acknowledge support from the EU through the ERC-starting grant ULPPIC.

\section{References:}

[1] J. Liu, X. Sun, R. Camacho-Aguilera, L.C. Kimerling, and J. Michel, "Ge-on-Si laser operating at room temperature," Opt. Lett., vol. 35, pp. 679-681, 2010.

[2] Chang-Hee Cho, Carlos O. Aspetti, Joohee Park, and Ritesh Agarwal, "Silicon coupled with plasmon nanocavities generates bright visible hot luminescence," Nature Photonics, vol. 7, pp. 285-289, 2013.

[3] X. Duan, Y. Huang, R. Agarwal, and C. M. Lieber, "Single-nanowire electrically driven lasers," Nature, vol. 421, pp. 241-245, 2003.

[4] T. A. Langdo, C. W. Leitz, M. T. Currie, E. A. Fitzgerald, A. Lochtefeld, and D. A. Antoniadis, "High quality Ge on Si by epitaxial necking," Appl. Phys. Lett., vol. 76, pp. 3700, 2000.

[5] R. Chen, T. Tran, K. Ng, W. Ko, L. Chuang, F. Sedgwick, and C. Chang-Hasnain, "Nanolasers grown on silicon,"Nature Photonics, vol. 5, pp. 170-175, 2011. 